\title{
A HIGHLY REDUNDANT ROBOT SYSTEM FOR INSPECTION
}

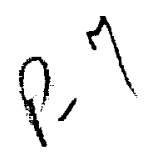

\author{
Thomas S. Lee, Tim Ohms, and Samad Hayati \\ lee@telerobotics.jpl.nasa.gov \\ Jet Propulsion Laboratory \\ California Institute of Technology \\ Pasadena, California, 91109
}

\begin{abstract}
The work on the serpentine inspection system at $=J P L$ is described. The configuration of the inspection system consists of 20 DOF in total. In particular, the design and development of the serpentine micromanipulator end-effector tool which has 12 DOF is described. The inspection system is used for application in JPL's Remote Surface Inspection project and as a research tool in redundant manipulator control.
\end{abstract}

\section{Introduction}

For several years, the Jet Propulsion Laboratory (JPL) has been performing research and development in remote surface inspection of space platforms such as Space Station Freedom [1]. One of our goals was to develop technology to inspect remote, hard-to-reach locations. Our experimental facility contains a $1 / 3$ sized mockup of the Space Station truss structure with various devices attached. The structure is cluttered with different types of objects such as an Orbital Replacement Unit (ORU) and a thermal radiator. The tasks to be performed range from visual inspection by maneuvering inside of narrowly confined areas and detecting anomalies to temperature and gas leak detection. One such scenario is moving behind a radiator panel and searching for electrical damages. Others include detection of broken interfaces such as disconnections in fluid, gas (leaks), or electrical lines and improper mating of connectors. There are some light manipulation tasks which are required to diagnose, service, and repair devices attached to the space structure. Some of the manipulation tasks include spot cleaning, foreign object debris location and removal, and removal/installation of straps and caps for lenses or containers.

Conventional robots typically consists of 6 Degreesof-Freedom (DOF), and are not capable of performing

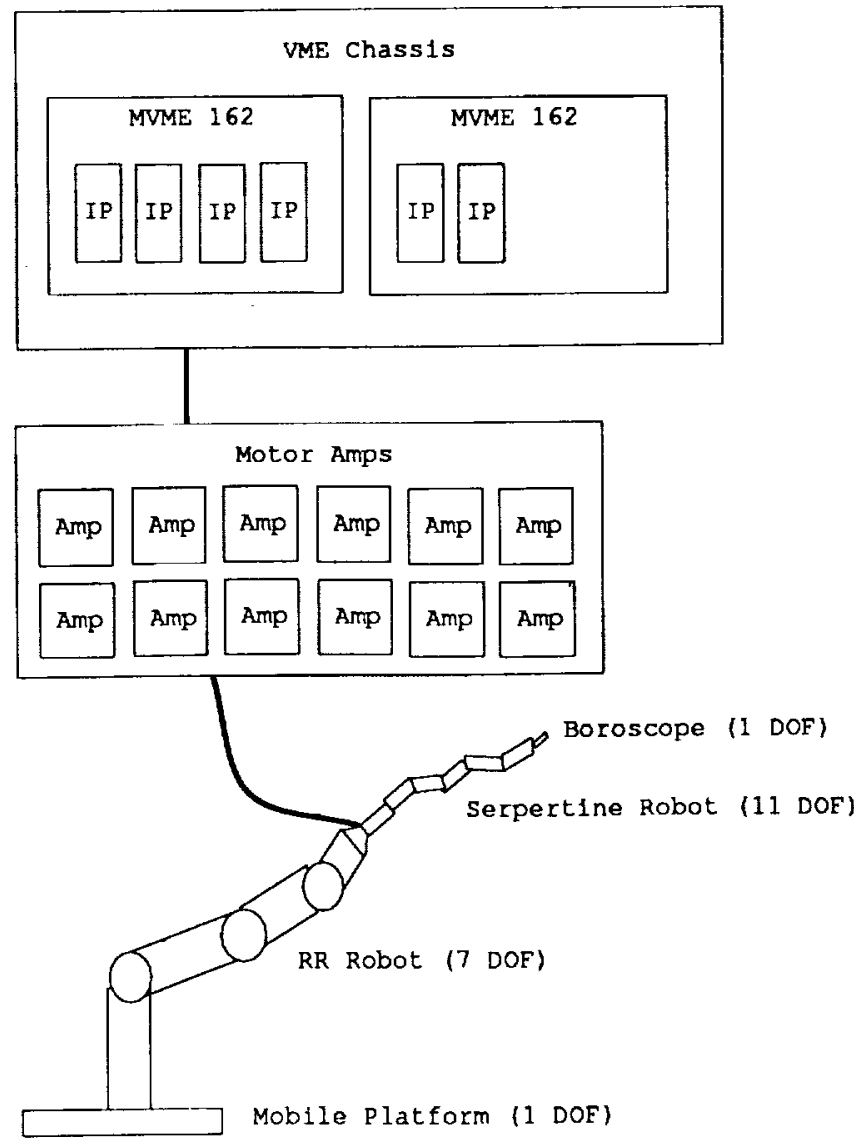

Figure 1: Overall Inspection System and the Hardware Architecture

some of the required remote inspection tasks. At JPL a highly redundant robot inspection system consisting of $20 \mathrm{DOF}$ will be utilized. The idea is to attach a smart end-effector tool that has a long-reach serpentine feature at the end of a conventional robot. This arrangement is referred as a compound robot - the serpentine robot is the micro-manipulator, and the base robot is the macro-manipulator. Figure 1 shows this configuration. Note that the 7 DOF of Robotics Re- 
search arm is mounted on a 1 DOF mobile base. The macro-manipulator can be thought of as a global positioning device, while the micro-manipulator can be viewed as a fine manipulator restricted to operate in a local region. In this paper, the design and development of the serpentine micro-manipulator is described. (see Figure 2).

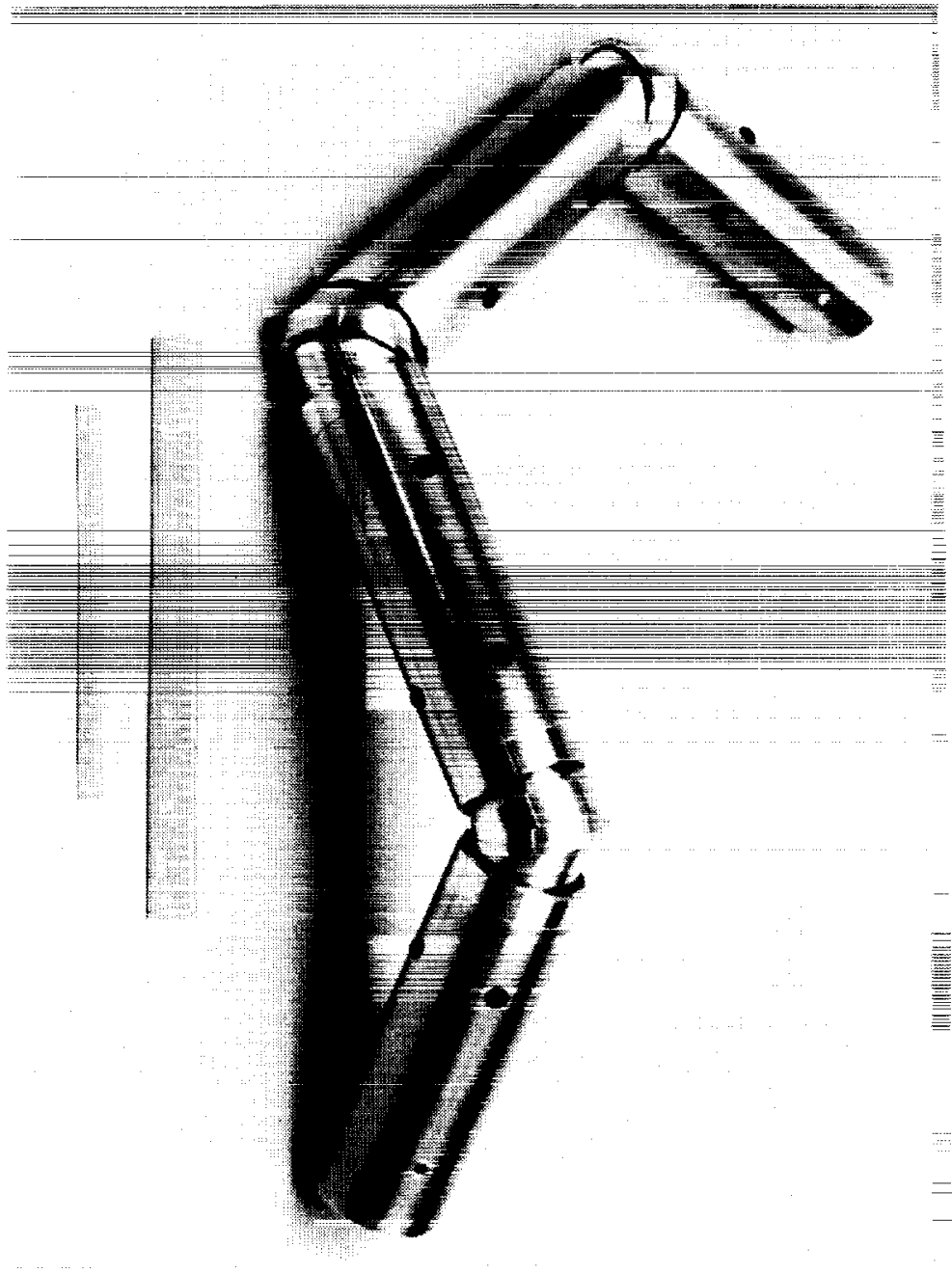

Figure 2: The JPL Serpentine Robot

\section{Background}

Work in serpentine robotics dates back approximately 30 years. Namely, the Japanese companies such as Toshiba, Mitsubishi, and Hitachi have done a lot of work in this area for application in the nuclear power industry. Hirose [2] of Tokyo Institute of Technology developed a number of snake-like mechanisms, for example, a crawling mechanism which utilizes oblique swivel joints. Asano [3] built Toshiba's
Self Approach System in 1982. A camera was mounted on the tip of this 16 DOF tendon-driven mechanism to perform inspection. In the Unites States, notable works include Anderson and Horn [5] who built a 16 DOF tensor arm for Scripps Institute of Oceanography in 1964. Chirikjian and Burdick [6] of Caltech built a 30 DOF variable geometry truss manipulator to validate hyper-redundant arm control algorithms. Berka [7] performed research in multi-segment robots for NASA's Johnson Space Center.

\section{Serpentine Robot Design}

At the end of the macro-manipulator, an integrated sensor/end-effector (ISEE) unit is attached [4]. It contains 2 lipstick cameras, 2 proximity sensors, a gas sensor, a temperature sensor, a force/torque sensor, and two light fixtures. This unit is too bulky to enter inside of the mockup truss structure. To overcome this restriction, a serpentine robot that can function as a smart end-effector tool was designed. The serpentine robot would be picked up by the macro-manipulator when additional dexterity is required to perform the task.

A number of design issues were considered before building the serpentine robot. The issues and their resolutions are discussed as follows.

\section{A. Weight and Size}

Since the serpentine robot is to be attached at the end of another robot, weight and size needed to be minimized.

Motor Selection: Miniature, yet high torque motors were needed. Motor manufacturers such as Escap, Maxon, and MicroMo were considered. MicroMo's 2 watt DC motors were chosen. Based on ironless core technology, these products have the feature of high efficiency with low mechanical time constants. The motors have stall current of $890 \mathrm{~mA}$, and due to their low inductance, electrical noise is reduced.

Joint Assembly: The joint design needed to be compact. If the conventional method of mounting the motors on the joints were adopted, the serpentine robot would have had a bulky design. A patented design owned by the NEC Corporation was chosen. This design allows all motors to be mounted inside of the joint housings.

The original design is an active universal joint based 


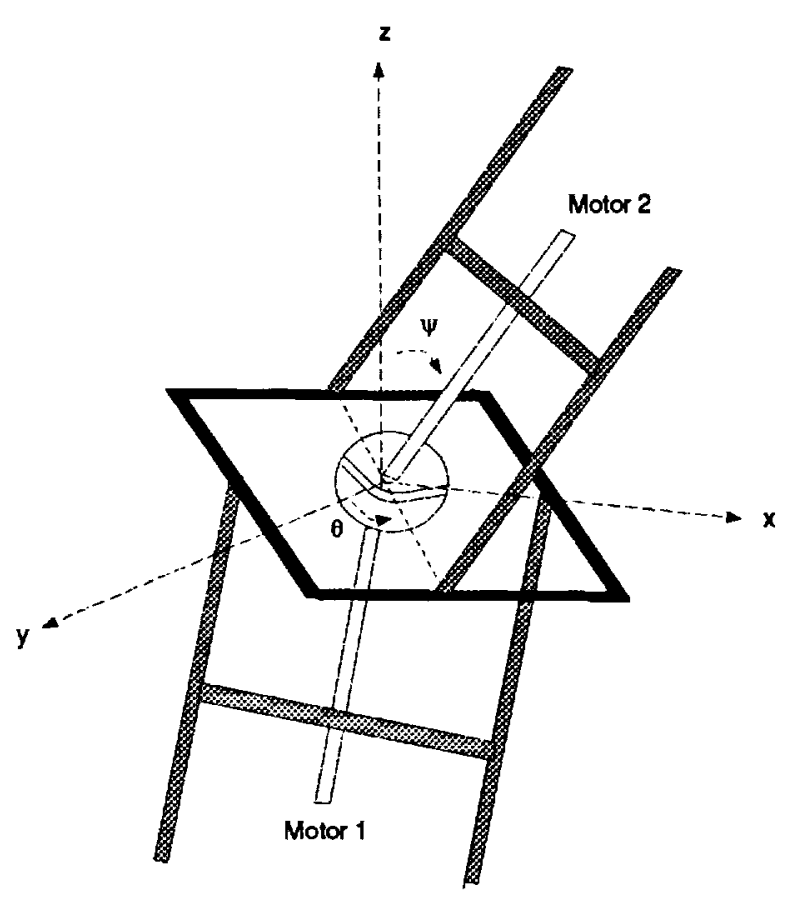

Figure 3: Joint Assembly

on work by Ikeda and Takanashi of the NEC Corporation (U.S. Patent No. 4,683,406). Our mechanism was made more compact by modifying their design. The basic idea is illustrated in Figure 3 . The joint assembly has two shafts, with each shaft attached to a half-sphere at an oblique angle. The two half-spheres are joined together to rotate freely with respect to each other. This arrangement is contained inside a universal joint with each shaft joined to one side of the frames that make up the universal joint. The motors rotate the two shafts thereby actively changing the orientation of the universal joint. Both motors are controlled simultaneously to change the orientation. Now consider the Spherical coordinate system. When the motors are rotated in the same circular directions, the joint assembly makes a motion along the $\psi$ direction. If the motors are rotated in opposite directions, then the joint assembly makes a motion along the $\theta$ direction. The motions along the $\psi$ and $\theta$ directions make up the 2 DOF movement of the joint. Note that when the shafts are collinear, a degeneracy (singularity) occurs.

To achieve high torque, each axis has a gearhead ratio of 1111:1 (high gear ratio was achieved by building our own custom planetary stages). Two redundant motors which are mechanically coupled turn each axis and provide double the torque of one motor. The gear- train is non-backdriveable for reduced power consumption. Maximum torque at each DOF was theoretically computed to be 90 in-lb, which was experimentally verified. Figure 4 and Figure 5 reveal the internals of the joint assembly.

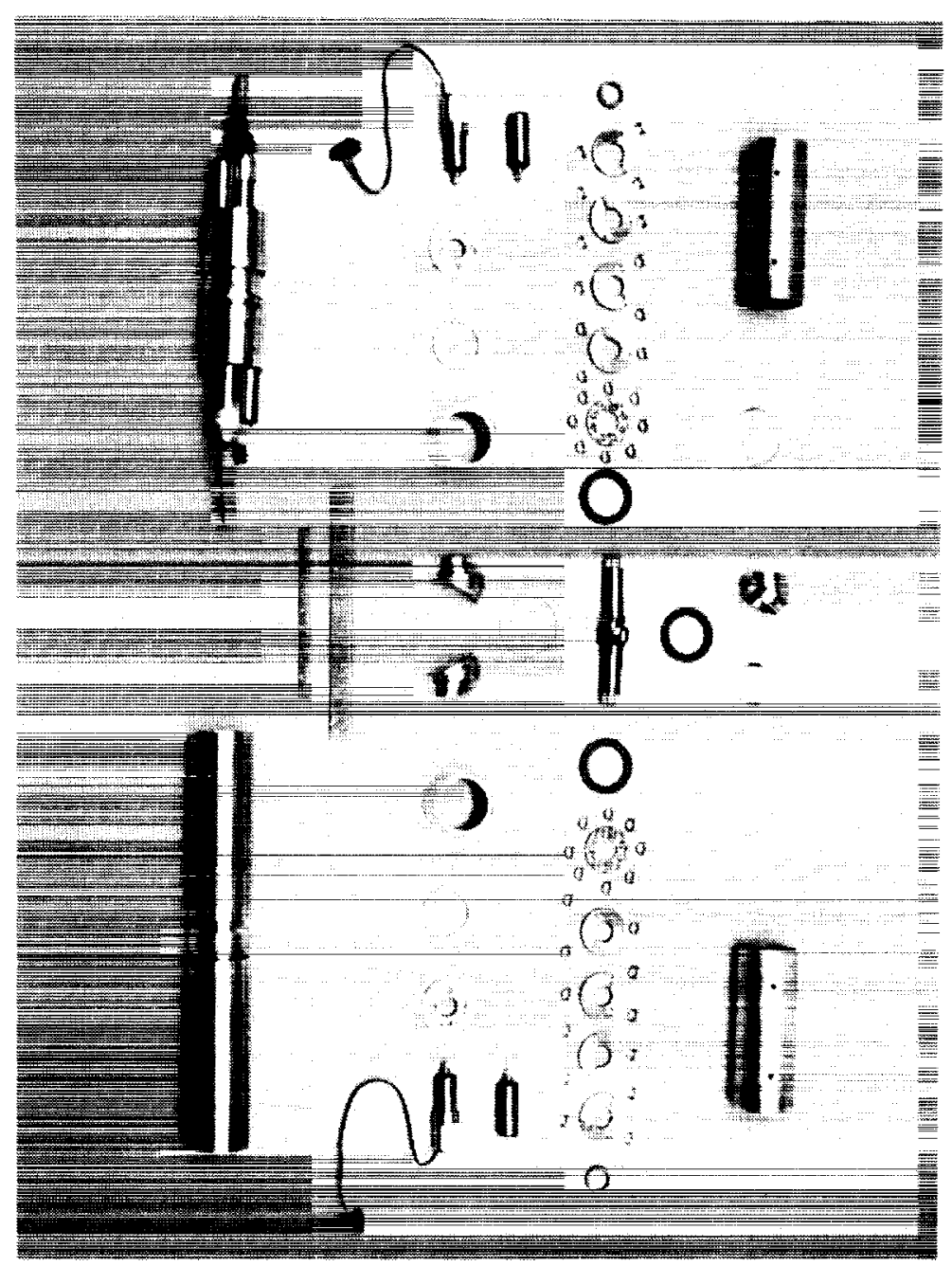

Figure 4: Components of the Serpentine Joint

\section{B. Reliability and Ease of Control}

To reduce the size and weight, building a tendondriven mechanism was considered. This approach is appealing because the actuators can be moved to the base of the serpentine robot. Since the entire serpentine robot including its base needed to be picked up by another robot, the overall mass is not saved by using this approach. In addition, inherent difficulties exist in dealing with a complicated tendon mechanism. This type of mechanism typically has a small load capacity, and it is difficult to model. Problems exist because of 


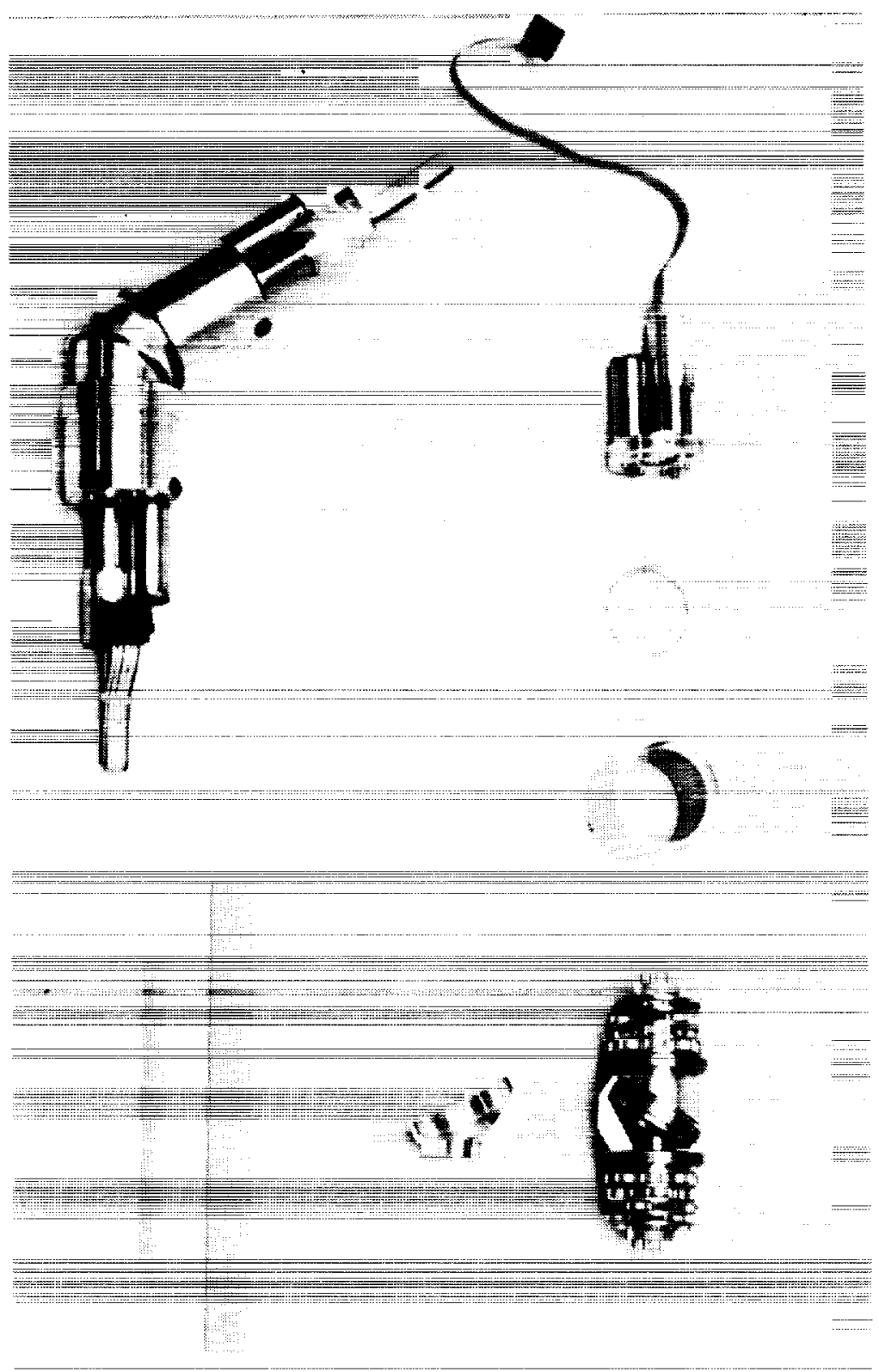

Figure 5: Internals of the Joint Assembly showing the Planetary Stages

the need for flexible control to compensate for elasticity. Finally, low reliability results due to frequent tendon breakage.

A method of direct motor control was chosen. Although the problems associated with high gear ratio will have to be dealt with, better reliability would be obtained.

\section{Modularity}

The mechanism was designed to be mechanically modular - the joints can be easily added or sub- tracted. The concern was more on the electrical side.

Designing miniature circuits to fit inside of the joint housing was considered. The electronics would provide the functionalities of a motor amplifier and a decoder for encoder signals. In designing a linear amplifier, elimination of heat generated by the electronics would create a problem since insufficient volume exists for air ventilation. Even a cooler PWM-based amplifier that employs miniature H-bridges could not be contained, since the size of all of its electronics would exceed the size of the joint housing (a cylinder of 1.5 inches in diameter with 5.65 inches height). To generate control signals, commerciallyavailable controllers such as the NEC uPD7832x, Hewlett Packard's HCTL-1100, and LM628 chips were considered. Circuit designs based on any of these chips would exceed the size of the joint housing.

The option to route all the wires out of the robot was chosen. The motors will be controlled remotely from externally located VME hardware. Routing all wires internally through the center hole posed another problem - cabling. Because 23 motors exist inside of the serpentine robot, the number of through-hole wires had to be minimized. The wire count was reduced at each DOF by connecting two motors in parallel to share motor voltage lines and by sharing common power lines for all motors. See Figure 6 for the wiring diagram.

For external VME control of the motors, off-theshelf hardware were purchased. Because of the motor's low inductance, linear analog amplifiers rather than PWM types were chosen as motor drives. Motor controller hardware were purchased to work in the VMEbus environment.

\section{Acquiring Visual Data and Lighting}

Mounting a small lipstick camera (e.g., Toshiba Model IK-M41A) at the tip of the serpentine robot was considered. This approach has associated problems with wiring and lighting. The diameter of the camera's cable far exceeds the size of the through-hole. Furthermore, the standard way of providing light for the camera is to resort to installation of light fixtures. But since the light fixtures are typically larger than the lipstick cameras, the size advantage of using the miniature cameras would be lost.

Using a borescope was ideal for our purpose. A borescope is designed specifically for visual inspection 
applications. It is commonly used in medical surgeries and aircraft engine inspections. The video image of the work site is passed through its fiberoptic cable and is sent remotely to the viewer - most of the vision hardware is located away from the robot's endeffector, hence moving the bulkiness away from the work site.

The Machida FBA-3-140 flexible borescope was chosen. The fiberoptic cable has a diameter of 0.138 inch $(3.5 \mathrm{~mm})$ and 55 inches long. With a throughhole of 0.312 inch $(5 / 16 \mathrm{inch})$, both the borescope cable and required control wires were routed internally. The scope has a field of view of 50 degrees minimum and a depth of field of 5 to $50 \mathrm{~mm}$.

The borescope is capable of 1 DOF motion. The tip is articulated by manually moving the lever at the eyepiece which pulls the cables attached to the tip. It is capable of a range of motion from -100 to 100 degrees. The function of the lever was motorized by installing a motor at the base of the serpentine robot to pull the cables. A working channel can be mounted along the side of the borescope to allow remote use of small tools, for instance, a grasping tool to retrieve foreign objects and a grinding tool to smooth surfaces. A working channel may be installed in the future to perform simple manipulation tasks.

An advantage of using a borescope is it carries its own light. When the serpentine robot enters the inside of the space structure, the environment is typically dark. Therefore, to acquire visual images, lighting is required. With the borescope, lighting is built into the cable and points in the same direction as the head of the borescope. Since our mockup structure composed mostly of metals with high reflectance, minimal light for the borescope was required - a Halogen light source served our purpose.

One drawback of using a borescope is it cannot by itself bore into the work area. A common way is simply pushing the borescope to insert it into the work area. To assist in the boring operation, for example in medical application, guide tubes are available to make possible insertion into difficult places where obstructions or large gaps exist. The guides are contouring apparatus to make angled turns possible by conforming to the desired insertion path. Here the serpentine robot can be thought of as a flexible guide tube for the borescope. The serpentine robot will act as a contoured platform for the borescope to rest on while the operator looks around the work area.

\section{E. Mechanical Specifications}

Constructed serpentine robot has the following specifications:

- 3-D Mechanism with Total Weight of $7 \mathrm{lbs}$

- Extended Reach: 35"

- Diameter of the Robot: $1.5 "$

- 5 Joints, 10 DOF (each $-60^{\circ}$ to $60^{\circ}$ )

- 1 Roll DOF $\left(-180^{\circ}\right.$ to $\left.180^{\circ}\right)$

- 1 Borescope DOF $\left(-100^{\circ}\right.$ to $\left.100^{\circ}\right)$

- DOF Velocity : 60 degrees/second

- Center-to-Center Joint Distance: 5.65"

- Through-Hole Inside for Cables: $5 / 16 "$

\section{F. Macro-manipulator}

The larger manipulator is the Robotics Research Corporation's Model K1207 robot which has 7 DOF. This arm is mounted on a mobile platform of the lathebed and provides one additional prismatic DOF. In total, 8 DOF comprise the macro-manipulator.

\section{G. User Interface}

The operator will interface with the serpentine robot from the "cupola," which is the main control station of the experimental facility of the Remote Surface Inspection project. Inside the cupola, one has access to an IRIS Silicon Graphics workstation, color monitors, and joysticks. The IRIS will act as a graphical front-end through which the operator interacts with the serpentine robot in real-time and issues motion commands in joint or task space. The IRIS can also create an interactive graphical simulation environment for analysis and control of the serpentine robot. Using this dual-mode functionality, the IRIS can be used in preview mode for animating the task scenario, followed by commanding the arm to duplicate the simulated motion.

The operator will view the work site by looking at the monitors that display video images from the borescope, and he will command the serpentine robot by using the joysticks and a graphical menu on the IRIS. 


\section{Serpentine Robot Control System}

Industry Pack (IP) Servo modules from Technology 80 , Inc. are used to control the motors in a VMEbus environment. These units are built around National Semiconductor's LM628 ICs and provide 2 independent channels for PID motor control and decoding of encoder signals. The IP-Servo modules are mounted on MVME162 Motorola processor boards which are based on the MC68040 hosts running at $25 \mathrm{MHz}$. See Figure 1 for the hardware architecture. To control the serpentine robot, two Motorola processor boards are employed to host six IP-Servo modules. The two processor boards are plugged into the same VME chassis that provide VME control for macro-manipulator system [8]. Through a shared memory card, command and status information of the serpentine robot are passed to the macro-manipulator system. All of the software executing on the VME environment is written in the $\mathrm{C}$ language. Code is developed on a SUN UNIX computer utilizing its resident $\mathrm{C}$ compiler and Wind River's VxWorks/Wind real-time library.

The IP-Servo module produces motor control signals in the form of voltages. The control signals are then taken as input to a linear analog amplifier. Portescap's ELD-3503 was chosen. This unit is a transconductance type of amplifier which is specifically designed to drive ironless motors. It produces up to 2.5 Amps of current and drives up to 35 Volt motors with a single DC power supply.

\section{Future Work}

In the near future, kinematic analysis will be performed to achieve Cartesian control of the serpentine system. In the process, a scheme to resolve redundancy of the mechanism would have to be devised to allow a task to be performed by allowing cooperation between the macro- and micro-manipulators. One possible scenario is to allow cooperation between the two manipulators to avoid obstacles by having each manipulator to executing a separate redundancy resolution scheme with a different objective function. Second, control experiments will be performed and any instability problems will be resolved. Problems associated with high gear ratios may exist, and instability may be attributed to the joint assembly since the joint angles are indirectly controlled by motor angles.

Many practical issues need to be dealt with before a three dimensional serpentine robot can be used for a teleoperation task. The manipulation task is difficult, since the operator is maneuvering the robot inside a narrow-spaced workspace and the objects that are of interest to him are often visually obstructed.

Sensors are crucial in helping the operator to perform inspection. The borescope inside of the serpentine robot will provide the main visual feedback to the operator. An additional camera can be attached to one of the intermediate links of the serpentine robot to provide the operator with a wider view of the work area from a different perspective. Other sensors such as proximity sensors can be used to detect and avoid obstacles.

The tip of the borescope should be placed such that it is jitter-free (statically stable) to take still images and to be optimally positioned for collision avoidance. In this scenario, the active perception problem of moving the cameras (sensors) would have to be examined to obtain more information about the environment as the task progresses.

The system requires a man-machine interface capability to control the motion of the micro- and macromanipulators collectively or individually, control the viewing angles attached to the serpentine robot, and ability to work with a world model of the environment for collision avoidance.

Knowledge-based systems can be integrated into the inspection system. In order to guide the serpentine robot, the computer can assist the operator in controlling the camera viewing and lighting angles. Once the operator selects an object/feature, the system can automatically adjust the camera viewing angle (aligning to the normal of the surface and to have the greatest visibility) as well as the lighting angle and intensity for the best view.

In addition, being preoccupied with a difficult teleoperation task at hand, the operator should not have to be concerned about kinematic anomalies such as singularities and joint limits. The operator needs only to specify the trajectory of the head of the serpentine robot; the trajectory of the rest of the body should be computed autonomously with some guidelines from the operator.

All of the above requirements can be incorporated into a global scheme to resolve the kinematic redundancies of the micro- and macro-manipulators.

\section{Acknowledgements}


The research described in this document was performed at the Jet Propulsion Laboratory, California Institute of Technology, under a contract with the National Aeronautics and Space Administration. We would like to thank Nobuaki Takanashi for providing the information on the patented mechanical design.

\section{References}

[1] Hayati, S., et. al, "Remote Surface Inspection System," Robotics and Autonomous Systems, Vol 11, No. 1, pp. 45-59, May 1993.

[2] Hirose, S. et. al., "Design and Control of a Mobile Robot with an Articulated Body", The International Journal of Robotics Research, Vol. 9, No. 2, April 1990.

[3] Asano, K., et al., "Multijoint Inspection Robot," IEEE Transaction on Industrial Electronics, Vol IE30, No. 3, pp. 277-281, 1983.

[4] Volpe, R., Balaram, J.B., "Technology Development for Robotic Surface Inspection," Proceedings of the AIAA/NASA Conference on Intelligent Robots for Factory, Field, Service, and Space, Houston, Texas, March 21-24, 1994.

[5] Anderson, V., Horn, R.C., "Tensor-arm Manipulator Design," ASME Transaction, Vol. 67-DE-57, pp. 1-12, 1967.

[6] Chirikjian, G., Burdick, J., et. al, "Kinematics of IIyper-redundant Robot Locomotion with Applications to Grasping," Proceedings of the IEEE International Conference on Robotics and Automation, 1991.

[7] Berka, R., "Development of a Large Space Robot: A Multi-Segment Approach," NASA Johnson Space Center Internal Publication.

[8] Seraji, H., Long, M., Lee, T., "Motion Control of 7-DOF Arms: The Configuration Control Approach," IEEE Transactions of Robotics and Automation, Vol. 9, No. 2, April 1993.

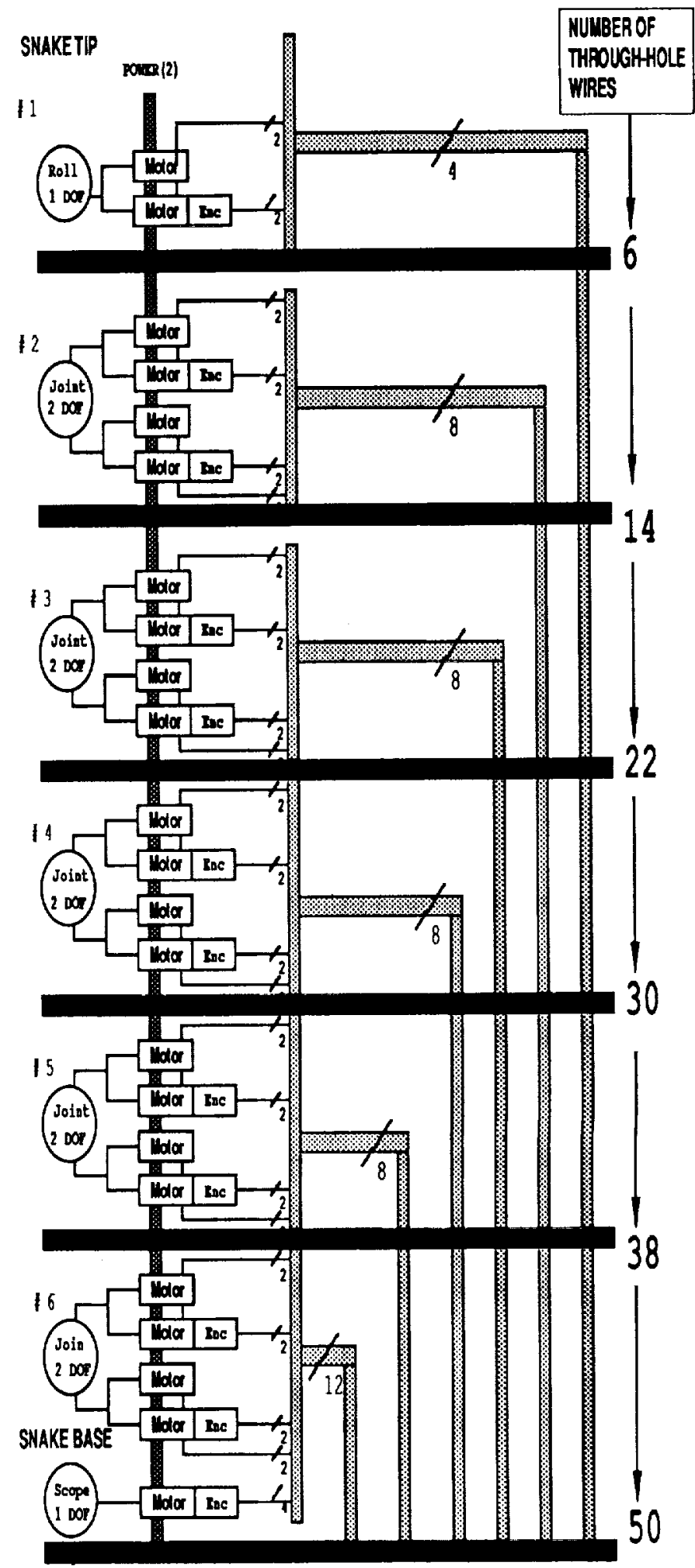

Figure 6: Wiring Diagram and the Serpentine Robot's DOF Distribution 